\title{
Efficient Spatial-Temporal Rebalancing of Shareable Bikes (Student Abstract)
}

\author{
Zichao Deng, ${ }^{1,2}$ Anqi Tu, ${ }^{1}$ Zelei Liu, ${ }^{1}$ Han Yu ${ }^{1}$ \\ ${ }^{1}$ School of Computer Science and Engineering, Nanyang Technological University (NTU), Singapore \\ ${ }^{2}$ Alibaba-NTU Singapore Joint Research Institute \\ \{zichao001, atu001\}@e.ntu.edu.sg, \{zelei.liu, han.yu\}@ntu.edu.sg
}

\begin{abstract}
Bike sharing systems are popular worldwide now. However, these systems are facing a problem - rebalancing of shareable bikes among different docking stations. To address this challenge, we propose an approach for the spatial-temporal rebalancing of shareable bikes which allows domain experts to optimize the rebalancing operation with their knowledge and preferences without relying on learning by trial-and-error.
\end{abstract}

\section{Introduction}

Bike sharing systems provide short-distance bike rental services for commuters with many docking stations scattering over an urban city. These systems bridge the gap between existing transportation modes and promote the efficiency of commuting in a sustainable way. However, due to dynamics of commuters' mobility, the bike supply/demand imbalance frequently occurs. It is important for system operators to rebalance bikes among docking stations and restore the number of bikes to its target value at each docking station by using trucks in an efficient and economical manner.

A common approach is to schedule batch rebalancing activities by trucks to move a larger number of bikes from docking stations with low demand to those with high demand (Li, Zheng, and Yang 2018). However, such trial-anderror-based approaches require long training time and may negatively impact user experience. In this paper, we propose the Spatial-Temporal Rebalancing (STR) algorithm for rebalancing shareable bikes over different periods in a day. Based on queueing system dynamics and Lyapunov drift, we formulate this task as a joint objective constrained optimization problem, which determines the number of bikes which should be moved into or out of each docking station at a given time slot to minimize both the time-averaged cost for moving bikes and the variation in the distribution of bikes among docking stations within the budget. A rebalancing schedule is then generated.

\section{The Proposed STR Approach}

A given shareable bike docking station $i$ can be modelled as a queueing system. The dynamics of $q_{i}(t)$ is as follows:

Copyright (C) 2020, Association for the Advancement of Artificial Intelligence (www.aaai.org). All rights reserved. $q_{i}(t+1)=\max \left[q_{i}(t)+\lambda_{i}(t)+a_{i}(t)-\mu_{i}(t), 0\right]$, where $q_{i}(t)$ is the number of bikes at docking station $i$ at time slot $t ; \lambda_{i}(t)$ is the number of incoming bikes to docking station $i$ at time slot $t$ (users dropping off bikes); $\mu_{i}(t)$ is the number of outgoing bikes from docking station $i$ at time slot $t$ (users picking up bikes); and $a_{i}(t)$ is the number of bikes which should be moved into or out of docking station $i$ at time slot $t$ by trucks as part of the bike rebalancing operation (i.e. the control variable in our problem).

We design the following Lyapunov function to model the distribution of bikes among docking stations: $L(t)=$ $\frac{1}{2} \sum_{i=1}^{N}\left[q_{i}(t)-\delta_{i}(t)\right]^{2}$, where $\delta_{i}(t) \geqslant 0$ denotes the target number of bikes at docking station $i$ at time slot $t$ in anticipation of demand in the near future. The time averaged conditional Lyapunov drift is expressed as: $\Delta=$ $\frac{1}{T} \sum_{t=0}^{T-1}\{L(t+1)-L(t) \mid \mathbf{q}(t)\}$. If normal usage results in large fluctuations in $\Delta$, rebalancing interventions need to be carried out in order to improve the operational efficiency.

The time-averaged cost for moving bikes between docking stations by trucks can be approximated as: $C=$ $\frac{1}{T} \sum_{t=0}^{T-1} \sum_{i=1}^{N} a_{i}(t) c(t)$. At different times of a day, the cost of moving bikes around by trucks may differ. This is mainly due to difference in road congestion levels. Overall, we aim to minimize a joint objective function of $\{$ cost+drift $\}$ which can be expressed as $\rho C+\Delta$, where $\rho>0$ is a weight factor a system operator can use to express his preference of cost saving over balancing demand and supply among bike docking stations. Therefore, the approach will minimize $f(t)=\frac{1}{T} \sum_{t=0}^{T-1} \sum_{i=1}^{N}\left\{\frac{1}{2} a_{i}^{2}(t)+a_{i}(t)\left[\rho c(t)+q_{i}(t)+\right.\right.$ $\left.\left.\lambda_{i}(t)-\mu_{i}(t)-\delta_{i}(t+1)\right]\right\}$, subject to $\sum_{i=1}^{N} a_{i}(t) c(t) \leqslant$ $B(t), \forall a_{i}(t)>0, \forall t$ where $B(t)$ is the total budget available for rebalancing bikes at time slot $t$.

By setting $\frac{d}{d a_{i}(t)} f(t)=0$, we have: $a_{i}(t)=\delta_{i}(t+1)+$ $\mu_{i}(t)-q_{i}(t)-\lambda_{i}(t)-\rho c(t)$. The intuition of the solution is "at a given point in time and a given docking station, if the predicted desirable bike stock level in the near future learned by the algorithm is high, the number of outgoing bikes is large, the current bike stock level is low, the number of incoming bikes is low, and the cost of moving bikes by truck is low, more bikes should be moved by truck to this docking station". 


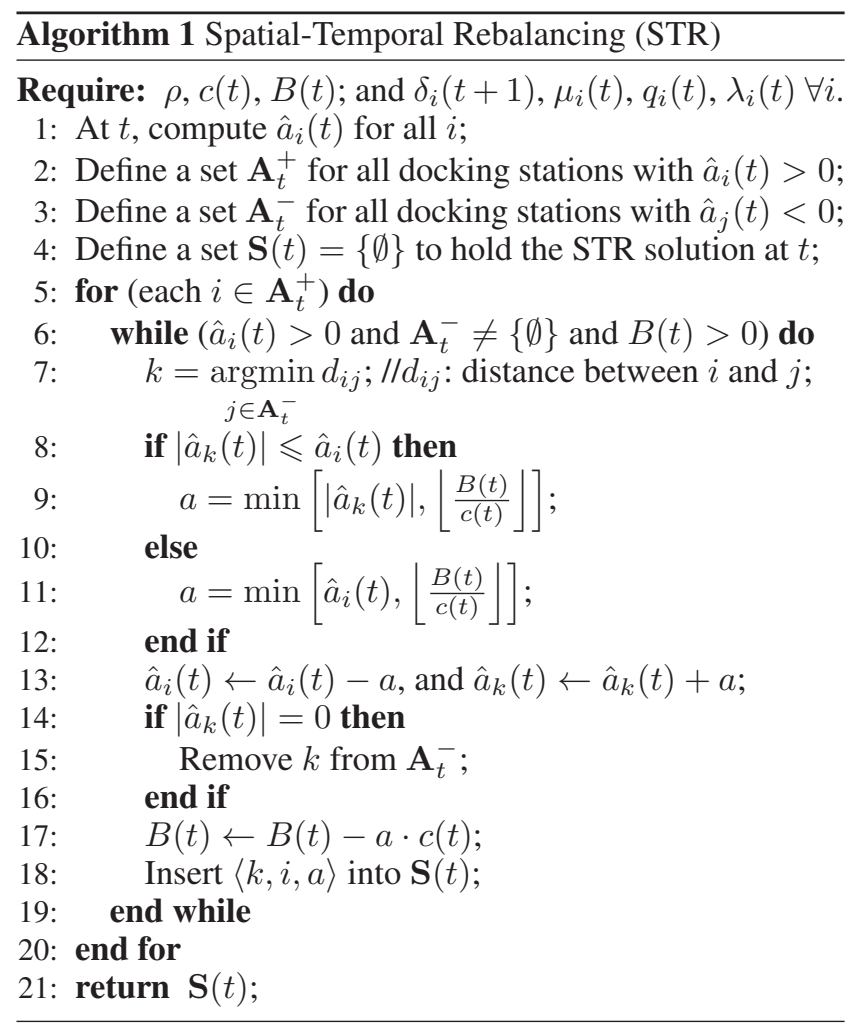

In this paper, we do not focus on computing $\delta_{i}(t+1)$, but delegate to existing short-term shareable bike demand forecasting models such as (Pan et al. 2019). We approximate $a_{i}(t)$ with the variable $\hat{a}_{i}(t)$ through a floor operator to ensure it is an integer: $\hat{a}_{i}(t)=\left\lfloor\delta_{i}(t+1)+\mu_{i}(t)-q_{i}(t)-\right.$ $\left.\lambda_{i}(t)-\rho c(t)\right\rfloor$. During a time slot $t$, if $\hat{a}_{i}(t)>0$, more bikes need to be moved into the docking station $i$; if $\hat{a}_{i}(t)<0$, $i$ can supply other docking stations with bikes; otherwise, $i$ should not participate in the bike rebalancing operation. Each entry into the bike rebalancing schedule is denoted as a tuple $\langle k, i, a\rangle$, which means that $a$ bikes are to be moved from docking station $k$ to docking station $i$ at the current time slot. This bike policy is implemented by Algorithm 1 .

\section{Results and Discussion}

In order to validate STR, we developed a simulator testbed (Figure 1) based on a real-world dataset from London containing a 36-day record of journeys in London bike sharing systems (https://www.kaggle.com/edenau/ london-bike-sharing-system-data). It simulates various conditions to study the performance of STR. A video demonstration can be found at https://youtu.be/OGj5z5_EH6A.

The shareable bike usage patterns and the number of bikes involved in STR rebalancing operations over a 24 hour period are shown in Figure 2(a). Two peak demand periods for shareable bikes in London are visible, one at around 9 to $10 \mathrm{am}$, the other around 6 to $8 \mathrm{pm}$. The peaks of rebalancing operations by STR occurred after the end of the morning peak usage period (to satisfy demand during early afternoon), and just before the evening peak period.

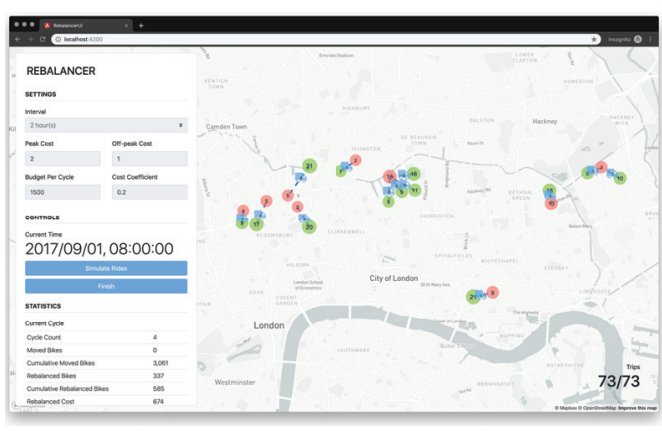

Figure 1: The STR simulation testbed.

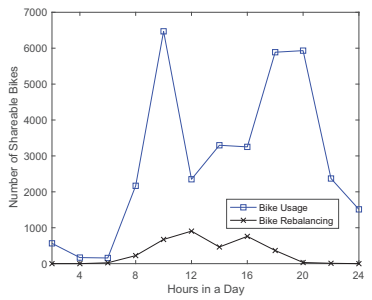

(a) Usage vs. Rebalance

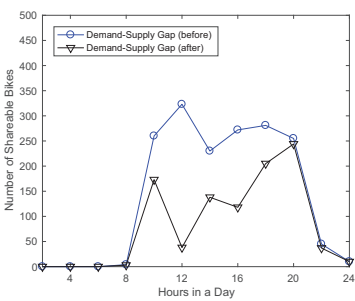

(b) Demand-Supply Gap
Figure 2: Experiment Results

Figure 2(b) shows the demand-supply gap across all docking stations before and after the STR rebalancing operations over a 24 hour period. It can be observed that during day time, STR significantly reduced the gap between demand and supply (in docking stations where demand outstrips supply). Towards the end of the evening peak period, STR stopped rebalancing as it expects that the demand-supply gap will disappear through normal usage towards midnight.

STR offers an effective mechanism for domain experts to transfer their knowledge and preferences to the AI algorithm, without having to rely on learning through trial-anderror. The resulting bike rebalancing plans can be readily explained to users to enhance transparency (Yu et al. 2018).

\section{Acknowledgements}

This research is supported by Nanyang Assistant Professorship (NAP), AISG-GC-2019-003, NRF-NRFI05-20190002, NTU-SDU-CFAIR (NSC-2019-011), and AlibabaNTU-AIR2019B1.

\section{References}

Li, Y.; Zheng, Y.; and Yang, Q. 2018. Dynamic bike reposition: A spatio-temporal reinforcement learning approach. In $K D D, 1724-1733$.

Pan, Y.; Zheng, R. C.; Zhang, J.; and Yao, X. 2019. Predicting bike sharing demand using recurrent neural networks. Procedia Computer Science 147:562-566.

Yu, H.; Shen, Z.; Miao, C.; Leung, C.; Lesser, V. R.; and Yang, Q. 2018. Building ethics into artificial intelligence. In IJCAI, 5527-5533. 